\title{
Total saponin from Anemone flaccida Fr. Schmidt abrogates osteoclast differentiation and bone resorption via the inhibition of RANKL-induced NF-KB, JNK and p38 MAPKs activation
}

\author{
Xiangying Kong ${ }^{1}$, Wenbin $\mathrm{Wu}^{1}$, Yue Yang ${ }^{1}$, Hongye Wan ${ }^{1}$, Xiaomin $\mathrm{Li}^{1}$, Michun Zhong ${ }^{1}$, Hongyan Zhao ${ }^{2}$,
} Xiaohui Su', Shiwei Jia ${ }^{3}$, Dahong Ju² and $\mathrm{Na}$ Lin $^{1 *}$

\begin{abstract}
Osteoclasts, bone-specialized multinucleated cells, are responsible for bone destructive diseases such as rheumatoid arthritis and osteoporosis. Natural plant-derived products have received substantial attention given their potential therapeutic and preventive activities against bone destructive diseases. In the present study, we investigated the effects of total saponin (TS) from Anemone flaccida Fr. Schmidt, on receptor activator of nuclear factor-kB ligand (RANKL)-induced in vitro osteoclast differentiation. We observed that TS concentration-dependently inhibited RANKL-induced osteoclast formation from RAW 264.7 cell and bone marrow-derived macrophages (BMMs), as well as decreased extent of actin ring formation and lacunar resorption. The RANKL-stimulated expression of osteoclast-related transcription factors were also diminished by TS. Moreover, TS blocked the RANKL-triggered TRAF6 expression, phosphorylation of mitogen-activated protein kinases (MAPKs) and IKB-a, and inhibited NF-kB p65 DNA binding activity. Furthermore, TS almost abrogated the nuclear factor of activated T cells (NFATc1) and c-Fos expression. Taken together, our results demonstrated that TS suppresses RANKL-induced osteoclast differentiation and inflammatory bone loss via the down-regulation of TRAF6 level, suppression of JNK and p38 MAPKs and NF-KB activation, and subsequent decreased expression of c-Fos and NFATC1. Therefore, TS may be a potential agent and needs to be more evaluated in vivo or in clinical trials to become a therapeutic for lytic bone diseases.
\end{abstract}

Keywords: Anemone flaccida, RANKL, Osteoclasts, Bone resorption, MAPKs, NF-KB

\section{Introduction}

Bone remodeling depends on the balance between bone resorption and bone formation [1]. It is necessary to repair damaged bone and to maintain mineral homeostasis. An imbalance in favor of bone resorption, most often due to excess osteoclastic activity, leads to bone loss in pathological conditions such as rheumatoid arthritis (RA) and osteoporosis [2]. Especially focal bone destruction within inflamed joints is the most specific hallmark of RA and leads to deformation, laxity, and functional disability [3].

\footnotetext{
* Correspondence: linna888@163.com

${ }^{1}$ Institute of Chinese Materia Medica, China Academy of Chinese Medical Sciences, No. 16, Nanxiaojie, Dongzhimennei, Beijing 100700, China

Full list of author information is available at the end of the article
}

Osteoclasts are the primary cells responsible for bone resorption and play crucial roles not only in maintaining bone homeostasis but also in development of pathological conditions such as joint destruction in RA [4-6]. Therefore, Osteoclasts become the main targets of current anti-resorptive drugs. The interaction of receptor activator of nuclear factor- $\mathrm{k} B$ ligand (RANKL) is essential for osteoclast differentiation and activation [7]. The binding of RANKL and RANK on osteoclast progenitor cells triggers the activation of tumor necrosis factor receptor-associated factor 6 (TRAF6) and subsequently the activation of NF- $\mathrm{BB}$ and mitogen-activated protein kinases (MAPKs), such as extracellular signalregulated kinase 1/2 (ERK1/2), p38 and c-Jun $\mathrm{N}$ terminal kinase (JNK) [8-10]. c-Fos and Nuclear factor 
of activated T cells (NFATc1) are two important downstream transcription factors in the RANKL/RANK signal pathway and play crucial role on the osteoclast differentiation $[9,11]$.

There is a growing interest in the utilization of medicinal plants for prevention and treatment of bone disorders including RA and osteoporosis [12]. The dry root of Anemone flaccida Fr. Schmidt, commonly known as "Di $\mathrm{Wu}$ " (Chinese name), is widely used as a folk medicine in the clinical compound prescription for the treatment of rheumatic diseases, external wounds and inflammations in China. Saponins are the characteristic components and also the main active ingredients of A. flaccida [13], and Diwu Fengshian Capsule with total saponin (TS) as the main active component has entered phase 3 clinical trial in China [14]. It has also been reported that saponins isolated from A. flaccida possess antiinflammation, immunoregulatory, analgesia and antitumor properties $[14,15]$. Bone metabolism is tightly associated with immune system and inflammation joint destruction in RA and other forms of arthropathies [16,17]. The antiinflammation and immune-regulatory properties of TS from A flaccida may prove its role in osteolytic bone diseases. However, to date the direct effects of $A$. flaccida on bone metabolism have not been studied.

In the present study, we investigated the direct effect of TS, the major bioactive constituents of A. flaccida, on osteoclast differentiation in RANKL-induced RAW 264.7 cells. Moreover, the possible mechanism associated with its inhibitory effect on osteoclast differentiation was also explored.

\section{Materials and methods Chemicals}

Alpha Modified Eagles Medium (a-MEM), fetal bovine serum (FBS), L-glutamine, penicillin and streptomycin, were purchased from Invitrogen Life Technologies. Mouse soluble RANKL was obtained from PeproTech Biotechnology. Antibodies against NFATc1 and TRAF6 were purchased from Santa Cruz Biotechnology. Antibodies against c-Fos, ERK, p-ERK, JNK, p-JNK, p38, p-p38, p65, p-IкB-a and GAPDH were obtained from Cell Signaling Technology. LightShift ${ }^{\mathrm{tm}}$ Chemiluminescent EMSA Kit was provided by Pierce Technology.

\section{Preparation of plant material and total saponins}

Rhizome of A. flaccida Fr. Schmidt was collected from Jiufeng County of Hubei Province, China. TS isolated from A. flaccida were provided by Dr. Shiwei Jia (GKH Pharmaceutical Ltd.) and prepared as reported $[13,15]$. TS were dissolved in dimethyl sulfoxide (DMSO) and diluted in PBS for storage in $-20^{\circ} \mathrm{C}$ freezer which is used in all subsequent experiments.

\section{Cell culture}

RAW 264.7 cells were obtained from the American Type Culture Collection (ATCC, Manassas, VA). RAW 264.7 cells were grown in a-MEM supplemented with $10 \%$ heat inactivated FBS, 2 mM L-glutamine and 100 $\mathrm{U} / \mathrm{ml}$ penicillin/streptomycin. Incubations were performed at $37^{\circ} \mathrm{C}$ in $5 \% \mathrm{CO} 2$, and cultures were fed every 2-3 days.

\section{Cell survival viability assay}

Cell viability was determined by 3-(4,5-dimethyl-2-thiazolyl)2,5-diphenyl-2H-tetrazolium bromide (MTS) method using CellTiter $96^{\circ}$ AQueous One Solution Cell Proliferation Assay (Promega, USA) according to the manufacturer's instructions. The experiments were carried out 3 times in triplicate measurements.

\section{Osteoclast formation}

RAW 264.7 cells were seeded onto a 96 well plate $\left(1 \times 10^{4}\right.$ cells/well $)$ with complete a-MEM containing RANKL (50 ng/mL) in the presence of TS or vehicle control for 6 days at $37^{\circ} \mathrm{C}$ and $5 \% \mathrm{CO}_{2}$. After 6 days, cells were washed with PBS and fixed with $10 \%$ formaldehyde for $15 \mathrm{~min}$. Then washed with PBS. Fixed cells were subjected to an assay for Tartrateresistant acid phosphatase (TRAP) activity. The experiments were carried out 3 times in triplicate measurements.

\section{Preparation of Bone Marrow-derived Macrophages (BMMs) and in vitro osteoclast formation}

Sprague -Dawley (SD) rats (4-7-week-old) were housed in Laboratory Animal Center (China Academy of Chinese Medical Sciences, Beijing, China) according to the guidelines for the care and use of laboratory animals of the NIH and China Academy of Chinese Medical Sciences (Beijing, China). All experimental procedures were approved by the Committee for Animal Use of the China Academy of Chinese Medical Sciences.

Total bone marrow cells were collected from tibia and femur of SD rat by flushing the marrow space with a-MEM. After removing the red blood cells (RBCs) with ACK buffer (0.01 mM EDTA, $0.011 \mathrm{M}$ KHCO3, and $0.155 \mathrm{M} \mathrm{NH} 4 \mathrm{Cl}, \mathrm{pH} 7.3$ ), cells were cultured for 1 day in a-MEM containing 10\% fetal bovine serum (FBS). Nonadherent cells were collected and further cultured with $20 \mathrm{ng} / \mathrm{mL}$ M-CSF in a-MEM containing $10 \%$ FBS. After 3 days, culture medium was removed and adherent cells were used for osteoclast differentiation. BMMs were cultured for 6 days in medium containing $20 \mathrm{ng} / \mathrm{mL} \mathrm{M}-\mathrm{CSF}$ and $50 \mathrm{ng} / \mathrm{mL}$ RANKL to induce osteoclast formation. 


\section{TRAP staining}

TRAP staining kit was obtained from Sigma Alrich (USA). TRAP staining was carried out according to the manufacturer's protocol. The images were taken with a digital camera attached to the microscope. TRAP positive multinucleated cells ( $\geq 3$ nuclei) were scored as osteoclastlike (OCL) cells. The number of TRAP-positive cells was counted using an eyepiece graticule at a magnification of 100 and the results expressed as the number of cells per field of vision.

\section{Immunofluorescent staining}

For F-actin ring staining, RAW 264.7 cells were stimulated with RANKL $(50 \mathrm{ng} / \mathrm{mL}$ ) for 6 days to induce osteoclast formation in the presence or absence of TS (0.1, 0.5 and $2.5 \mu \mathrm{g} / \mathrm{mL})$. For NF-kB-p65 nuclear translocation experiment, RAW 264.7 cells were pretreated in the presence of TS or vehicle control overnight, then added RANKL (50 ng/mL) for $30 \mathrm{~min}$. After incubation, the cultures were fixed in $4 \%$ paraformaldehyde in PBS and cells permeabilized with $0.1 \%$ Triton X-100 in PBS for $15 \mathrm{~min}$. Cells were then stained for F-actin by incubation in $1 \mathrm{mg} / \mathrm{ml}$ TRITC-conjugated phalloidin or by antiNF- $\mathrm{kB}-\mathrm{p} 65$ for $2 \mathrm{~h}$ at $37^{\circ} \mathrm{C}$. The preparations were then washed and mounted in Vectashield mounting medium with Hoechest 33258 (Vector Laboratories, Peterborough, UK). Cells were inspected with an Olympus x41 microscope. The experiments were carried out 3 times in triplicate measurements.

\section{Bone resorption pit assay}

To study the effect of TS on osteoclastic bone resorption, RAW 264.7 cells were seeded onto 50 um thick bovine bone slices and cultured with complete a-MEM containing RANKL (50 ng/mL) in the presence of TS or vehicle control at $37^{\circ} \mathrm{C}$ and $5 \% \mathrm{CO}_{2}$. After 7 days, cells were then removed by sonication and the bovine bone slices were stained with toluidine blue to identify resorption pits. Also resorption lacunae were visualized by a Hitachi S-3400 N scanning electron microscope.

\section{Enzyme Linked Immunosorbent Assay (ELISA)}

RAW 264.7 cells were suspended in a-MEM containing $10 \%$ FBS and plated at a concentration of $1 \times 10^{5}$ cells/ well into a 24-well culture dish (Corning). Then, they were treated with either TS or vehicle for $24 \mathrm{~h}$ before adding RANKL (50 ng/mL) to stimulate TNF-a secretion for $16 \mathrm{~h}$. At the end of culture, medium was collected and analyzed for TNF-a using the ELISA kit according to the manufacturer's instructions.

\section{Western blotting}

RAW 264.7 cells were seeded onto $75 \mathrm{~cm}^{2}$ flask pretreated with TS or vehicle control overnight at $37^{\circ} \mathrm{C}$ and
5\% CO2. Then RANKL (50 ng/mL) was added for 30 min. Western blot was performed according to our previously described protocol [18]. The primary antibodies used in the experiment included anti-ERK1/2(1:1000), anti-phospho-ERK1/2 (1:200), anti-Jun N-terminal kinase (JNK) (1:200), anti-phospho-JNK(1:200), anti-p38(1:500), anti-phospho-p38(1:200), anti-IкB $\alpha-p$ (1:250), anti-c-Fos (1:1000), anti-TRAF6 (1:1000), anti-GAPDH (1:1000). Horseradish peroxidase (HRP)-conjugated anti-mouse and anti-rabbit antibodies (1:5000) were used as secondary antibodies. The membranes were then visualized using the ECL system (Pierce, USA). GAPDH (internal control) was used to confirm equal protein loading.

\section{Electrophoretic Mobility Shift Assay (EMSA)}

RAW 264.7 cells were incubated with or without various concentrations of TS $(0.1,0.5,2.5 \mu \mathrm{g} / \mathrm{mL})$ for $12 \mathrm{~h}$. The cells were then treated with RANKL (50 ng/mL) for 30 min. The nuclear protein extracted from the cells using a NE-PER Nuclear and Cytoplasmic Extraction Reagent Kit (Pierce, USA). EMSA were performed according to protocol of the LightShiftTM Chemiluminescent EMSA Kit. The synthetic biotinlabeled double-stranded NF- $\kappa B$ consensus oligonucleotide (5'-AGTTGAGGGGACTTT CCCAGGC-3') were used to measure the effect of the TS on NF- $\mathrm{kB}$ nuclear protein-DNA binding activity. Briefly, nuclear proteins $(30 \mu \mathrm{g})$, poly $(\mathrm{dI}-\mathrm{dC})$, and biotin-labeled double-stranded NF- $\mathrm{kB}$ oligonucleotide were mixed with the binding buffer. In addition, the excess amounts of unlabeled oligonucleotide were used for the competition assay to confirm specificity of binding. The DNA/protein complex was electrophoresed on $5 \%$ non-denaturing polyacrylamide gel. The DNA mobility shift due to binding of NF- $\mathrm{kB}$ complex was detected with enhanced chemiluminescence (ECL) reagent (Pierce, USA).

\section{Statistical analysis}

All raw data were processed by authorized software SPSS 13.0. The data were analyzed by One-way ANOVA followed by Dunnett's t-test to assess the statistical significance of the differences between the study groups. Differences were considered statistically significant when $P$ was less than 0.05 .

\section{Results}

TS suppresses RANKL-induced osteoclast differentiation Since RAW 264.7 macrophage-like cells can differentiate into OCL cells as described elsewhere [19], the effect of TS on RANKL-induced osteoclast formation was examined using these cells. RAW 264.7 cells cultured in the presence of RANKL form multinucleated TRAP-positive OCL cells (Figure 1A). Addition of TS $(0.1-2.5 \mu \mathrm{g} / \mathrm{mL})$ into RAW 264.7 cells cultures showed dose-dependent 


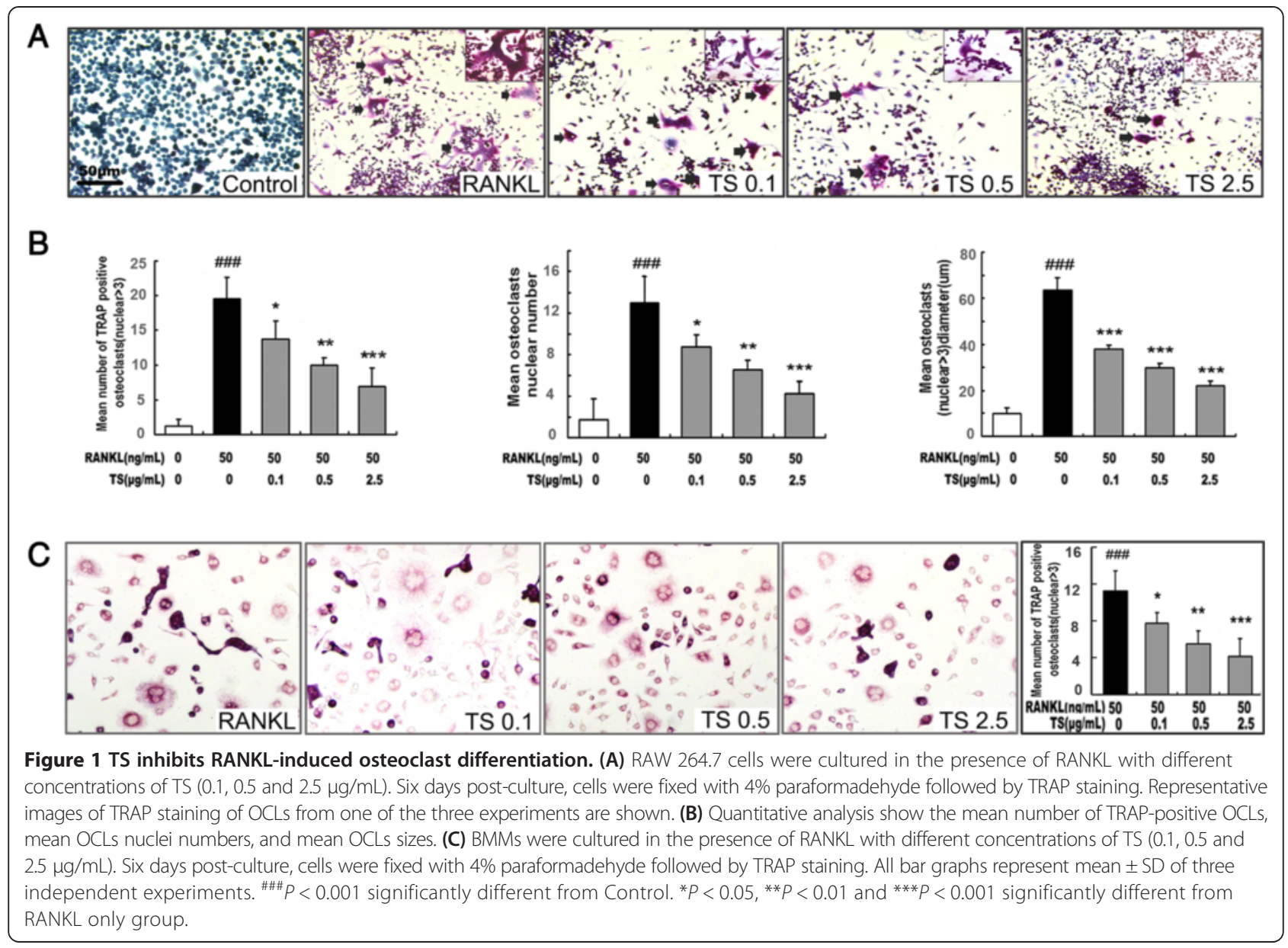

decrease the number of TRAP positive multinucleated cells (Figure 1A and B). Notably, OCL cells in cultures that were treated with TS exhibited morphological differences with control OCL cells, being smaller in size and containing fewer numbers of nuclei (Figure 1A and B).

The effects of TS were further confirmed using primary BMMs. TS inhibited the multinucleated osteoclast formation induced by RANKL and M-CSF in BMMs (Figure 1C).

\section{TS attenuates RANKL-stimulated F-actin rings formation and osteoclastic bone resorption}

To examine the effects of TS on osteoclastic bone resorption, equal numbers of RAW 264.7 cells were seeded onto bovine bone slices and thereafter TS was added to the culture. Bone surfaces were retrieved after incubation for 6 days, processed for toluidine blue staining or scanning electron microscopy, visualised and scored for bone resorption as described in the methods. Treatment of cultures with TS $(0.1-2.5 \mu \mathrm{g} / \mathrm{mL})$ can significantly attenuate osteoclastic bone resorption (Figure 2). Note that treatment of TS resulted in very shallower resorption pits or reduced pit areas as compared to untreated control both by toluidine blue staining or scanning electron microscopy (Figure 2A,B,D and $\mathrm{E}$ ).

The bone resorption function of osteoclasts depends on dynamic regulation of the actin cytoskeleton. Actin ring structure is a characteristic cytoskeletal feature of functional osteoclasts [20]. Therefore, we next examined whether TS affects actin ring structure of mature osteoclasts. OCLs were double-stained with phallotoxins and Hochest 33258 to allow for visualization of the cytoskeleton and nuclei respectively. In mature osteoclasts on tissue culture plates, F-actin was arranged into a ringlike structure (actin ring) at the cell periphery. Treatment of with TS $(0.1-2.5 \mu \mathrm{g} / \mathrm{mL})$ caused both shrinkage of osteoclasts and disruption of actin ring structure in a dose-dependent manner (Figure $2 \mathrm{C}$ and $\mathrm{F}$ ).

To examine whether the above suppressive effect of TS was due to its cytotoxicity, MTS assays were carried out to test cell survival viability. As shown in Figure $2 \mathrm{G}$ and Additional file 1: Figure S1, TS from 0.1 to $2.5 \mu \mathrm{g} / \mathrm{mL}$ did not shown any cytotoxicity on RAW 264.7 cells and 


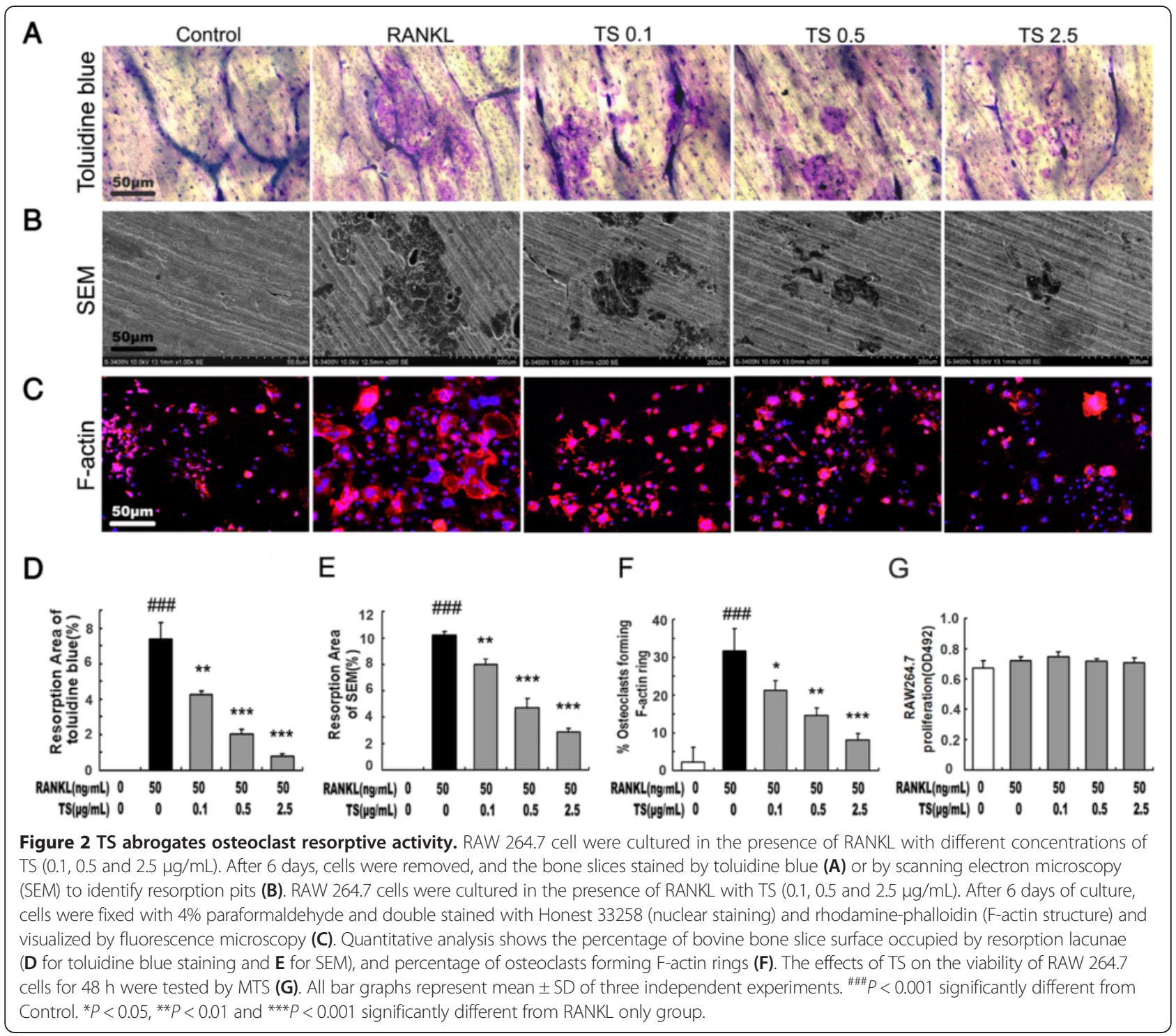

BMMs under the experimental conditions used in the present studies. No cytotoxicity of TS was also confirmed by a trypan blue dye exclusion test (Additional file 2: Figure S2). Furthermore, apoptosis of RAW 264.7 was also unaffected by these anti-osteoclastic TS concentrations (Additional file 3: Figure S3), suggesting that the observed reduction of osteoclast formation and activity was a result of osteoclast differentiation inhibition by TS.

\section{TS inhibits RANKL-stimulated secretion of inflammatory cytokines}

Inflammatory cytokines, such as TNF-a, induces osteoclasts formation, and stimulates osteoclastic bone resorption [21]. The effects of TS on the production of TNF-a induced by RANKL were studied on RAW 264.7 cells in vitro. As shown in Figure 3, RAW 264.7 cells produced a large quantity of TNF-a in response to RANKL stimulation. Treatment of RAW 264.7 cells with TS (0.1-2.5 $\mu \mathrm{g} / \mathrm{mL})$ effectively suppressed TNF- $\alpha$ production.

\section{TS abrogates RANKL-induced TRAF6 expression}

The binding of RANKL with its receptor RANK interacts and induces the trimerization and activation of signaling adaptor molecule TRAF6, which is an essential initiating step during osteoclast differentiation [9]. We first analyzed the possible action of TS on TRAF6 protein expression by Western blot. As shown in Figure 4A, RANKL (50 ng/mL) stimulation obviously up-regulated the expression of TRAF6 in RAW 264.7 cells, reaching peak accumulation at $24 \mathrm{~h}$. Therefore, in this study, we selected the induction time for $24 \mathrm{~h}$ to explore the effects of TS on TRAF6 protein expression. We found that 


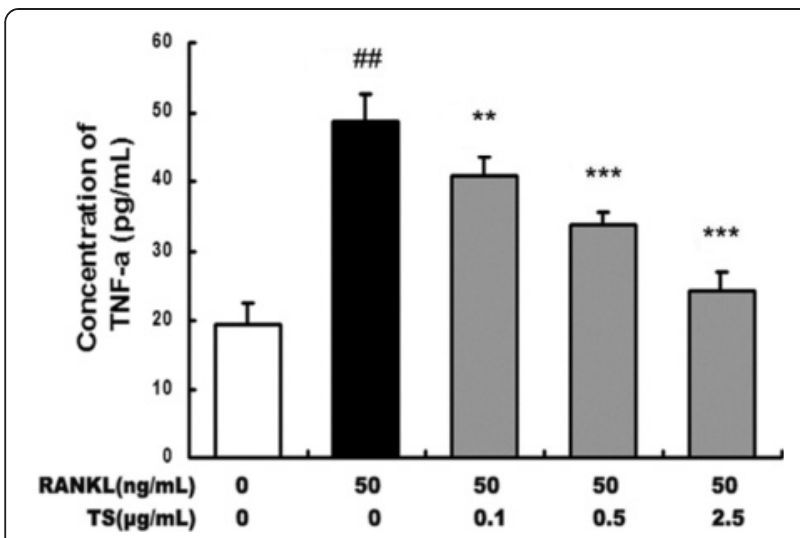

Figure 3 TS inhibits RANKL-induced TNF- $a$ s secreted by RAW 264.7 cells. Confluent RAW 264.7 cells were incubated with or without TS $(0.1,0.5$ and $2.5 \mu \mathrm{g} / \mathrm{mL})$ for $2 \mathrm{~h}$, followed by stimulation with RANKL $(50 \mathrm{ng} / \mathrm{mL})$ for $24 \mathrm{~h}$, and supernatants were analyzed for TNF-a by enzyme-linked immunosorbnent assay (ELISA). Data are represented as the mean \pm SD of three independent experiments. ${ }^{\# \#} P<0.001$ significantly different from control. ${ }^{* *} P<0.01$ and ${ }^{* *} P$ $<0.001$ significantly different from RANKL only group.

TS $(0.1,0.5$ and $2.5 \mu \mathrm{g} / \mathrm{mL})$ pre-treatments markedly inhibited RANKL-stimulated expression of TRAF6 (Figure 4B).

\section{TS inhibits RANKL-induced MAPKs phosphorylation}

MAPKs (mainly including ERK, JNK and p38 MAPK) are located at the downstream of the TRAF6 signalling complexes, and play an important role in RANKLinduced osteoclast differentiation. To further explore pathways by which TS regulates osteoclast differentiation and function, the effect of TS on RANKL-induced ERK, JNK and p38 phosphorylation was examined in RAW 264.7 cells. RANKL strongly activated ERK, JNK and p38 phosphorylation in RAW 264.7, whereas RANKL-induced phosphorylation was inhibited by TS $(0.1-2.5 \mu \mathrm{g} / \mathrm{mL})$, most effectively for JNK, less for p38, and nearly no influence on ERK (Figure 5). These results indicated that the mechanism for the anti-osteoclast differentiation effects of TS involved the inhibition of JNK and p38 MAPKs.

\section{TS suppresses RANKL-induced Activation of NF-KB signaling pathway}

RANKL-induced NF- $\mathrm{kB}$ activation is essential in initiating osteoclast differentiation. To determine whether TS inhibited NF-kB-mediated osteoclast differentiation, we investigated NF- $\mathrm{kB}$ activation in RAW 264.7 cells affected by TS. As shown in Figure 6A, thirty minutes of RANKL (50 ng/mL) treatment alone, induced an increase in IKB-a phosphorylation, and addition of TS resulted in a marked suppression of IkB-a phosphorylation (Figure 6A). To add to this, we investigated the effect of TS on nuclear translocation of the NF-kB-p65 subunit in RAW 264.7 cells. Pre-treatment $(2 \mathrm{~h})$ of cells with TS $(0.1,0.5$ and $2.5 \mu \mathrm{g} / \mathrm{mL})$ prior to RANKL stimulation $(0.5 \mathrm{~h})$ resulted in a significant reduction in NF-kB-p65 nuclear translocation in RAW 264.7 cells, which was evidenced by the reduced amounts in the nucleus (Figure 6B). Further the NF-kB DNA binding activity was investigated in the activated RAW 264.7 cells. As shown in Figure 6C, the nuclear extract from RANKL-stimulated RAW 264.7 cells showed a marked increase in NF- $\mathrm{kB}$ nuclear protein DNA-binding activity compared with the control. The specificity of the NF- $\kappa B$ nuclear protein-DNA binding was verified by competition assay with a 200-fold excess of unlabeled NF- $\mathrm{KB}$ probe. Pretreatment of cells with various concentrations of TS suppressed the activation of NF- $\mathrm{kB}$ binding to its consensus DNA sequences. Taken together, these results indicated that the inhibition of the NF- $\mathrm{BB}$ pathway by TS contributed to its suppression of osteoclast formation.
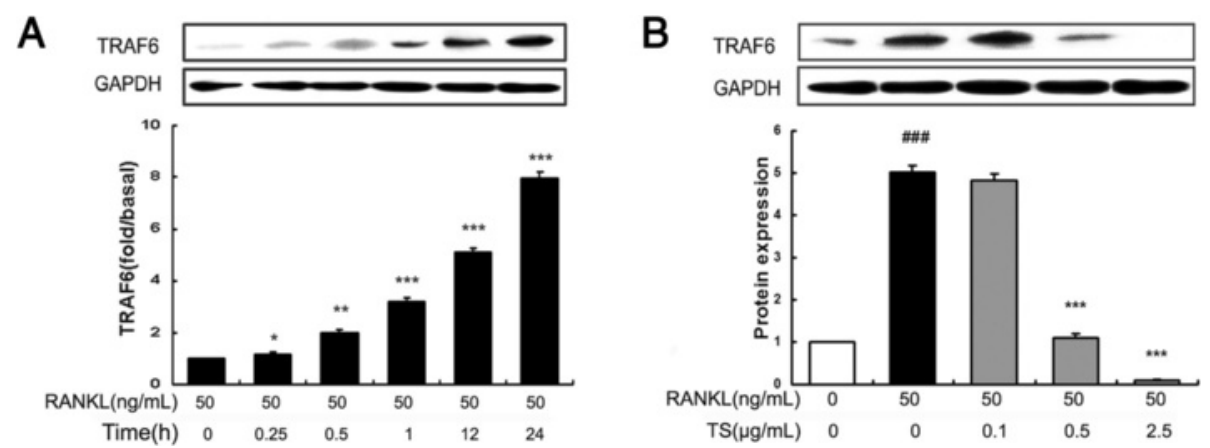

Figure 4 TS abrogates expression of TRAF6 in RANKL-induced RAW 264.7 cells. RAW 264.7 cells were pre-treated with RANKL (50 ng/mL), and cell lysates were collected at the time points indicated (A) and/or various concentrations of TS (0.1, $0.5 \mathrm{and} 2.5 \mu \mathrm{g} / \mathrm{mL})$ for $24 \mathrm{~h}$. The harvested cells from triplicate tests were subjected to western blot analysis for TRAF6 (B). Relative amount of protein were determined by densitometric analysis. One of three experiments with similar results is shown. Data are represented as the mean \pm SD. ${ }^{\# \#} P<0.001$ significantly different from control. ${ }^{*} P<0.05,{ }^{* *} P<0.01$ and ${ }^{* *} P<0.001$ significantly different from RANKL only group. 


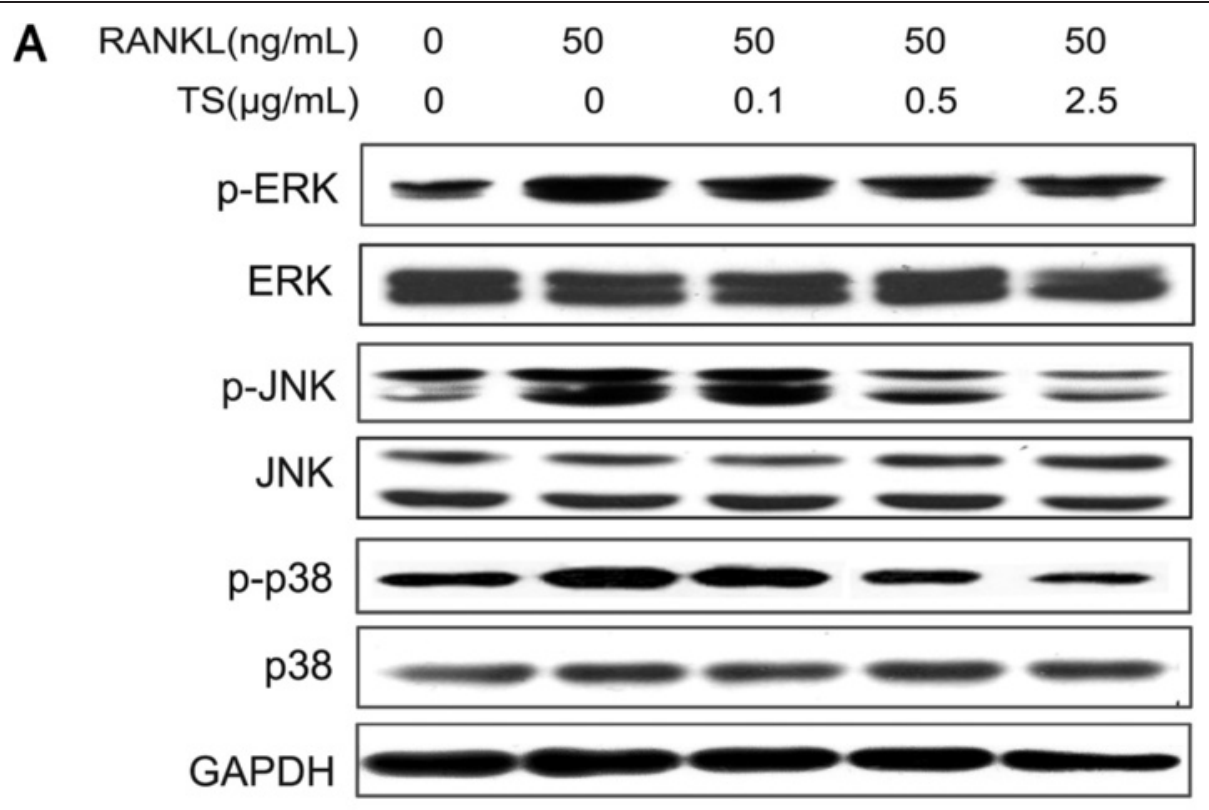

B

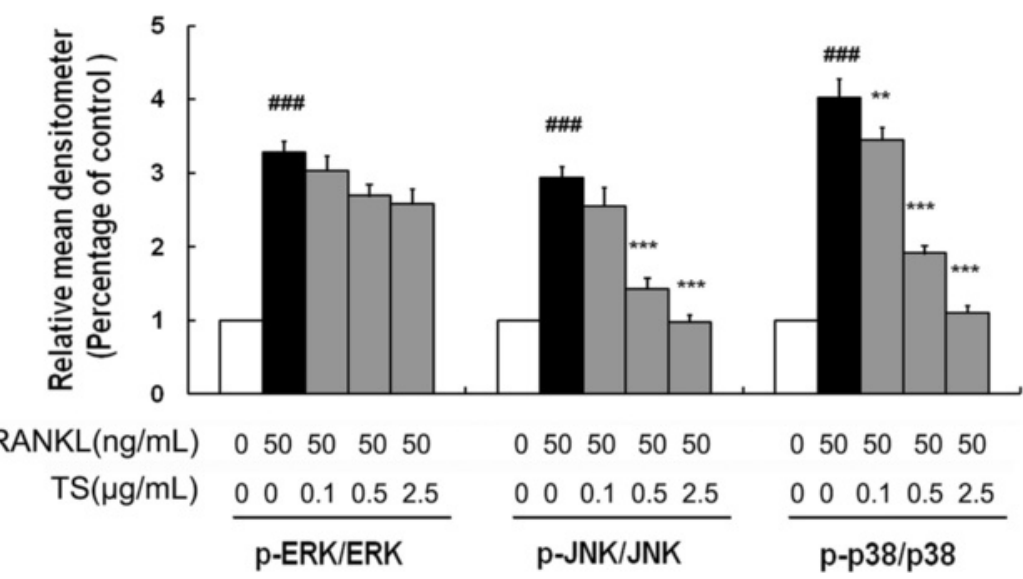

Figure 5 TS attenuates JNK and p38 MAPKs activation in RANKL-induced RAW 264.7 cells. RAW 264.7 cells were preincubated with the indicated concentrations of TS $(0.1,0.5$ and $2.5 \mu \mathrm{g} / \mathrm{mL})$ for $2 \mathrm{~h}$, and then treated with $50 \mathrm{ng} / \mathrm{mL}$ RANKL for $30 \mathrm{~min}$. Total protein was extracted and subjected to Western blot analysis (A). For phosphorylated proteins, the ratio of phosphorylated to total species was determined by densitometry and normalization to glyceraldehyde 3-phosphate dehydrogenase (GAPDH) levels (B). One of three experiments with similar results is shown. Data are represented as the mean \pm SD three independent experiments. ${ }^{\# \#} P<0.001$ significantly different from control. ${ }^{* *} P<0.01$ and ${ }^{* * *} P<0.001$ significantly different from RANKL only group.

TS down-regulates RANKL-induced expression of NFATc1 and c-Fos

Binding of RANKL to RANK should activate several transcription factors that are responsible for promoting osteoclastic gene expression. NFATc1 and c-Fos are identified as two of the most important osteoclasts specific transcription factors [22]. As shown in Figure 7A and B, RANKL stimulation for $24 \mathrm{~h}$ obviously up-regulated the expression of both c-Fos and NFATc1 in RAW 264.7 cells. Additionally, TS $(0.1,0.5$ and $2.5 \mu \mathrm{g} / \mathrm{mL})$ treatments markedly inhibited the expression of c-Fos and NFATc1 with the concentration dependent manner.

\section{Discussion}

The root of $A$. flaccida has long been used for the treatment of arthritis and traumatic injury in folk medicine of China. TS is the main active constituent of this herb medicine and has been previously demonstrated to exhibit beneficial effects on adjuvant-induced arthritis by its immunomodulatory and anti-inflammation effects $[14,23]$. However, the effects of TS on the bone resorption remain unclear. The results in this study showed that TS inhibits osteoclast formation and bone resorption via the suppression of RANKL-induced activation of NF- $\mathrm{KB}$ and MAPKs signaling pathway. These data 


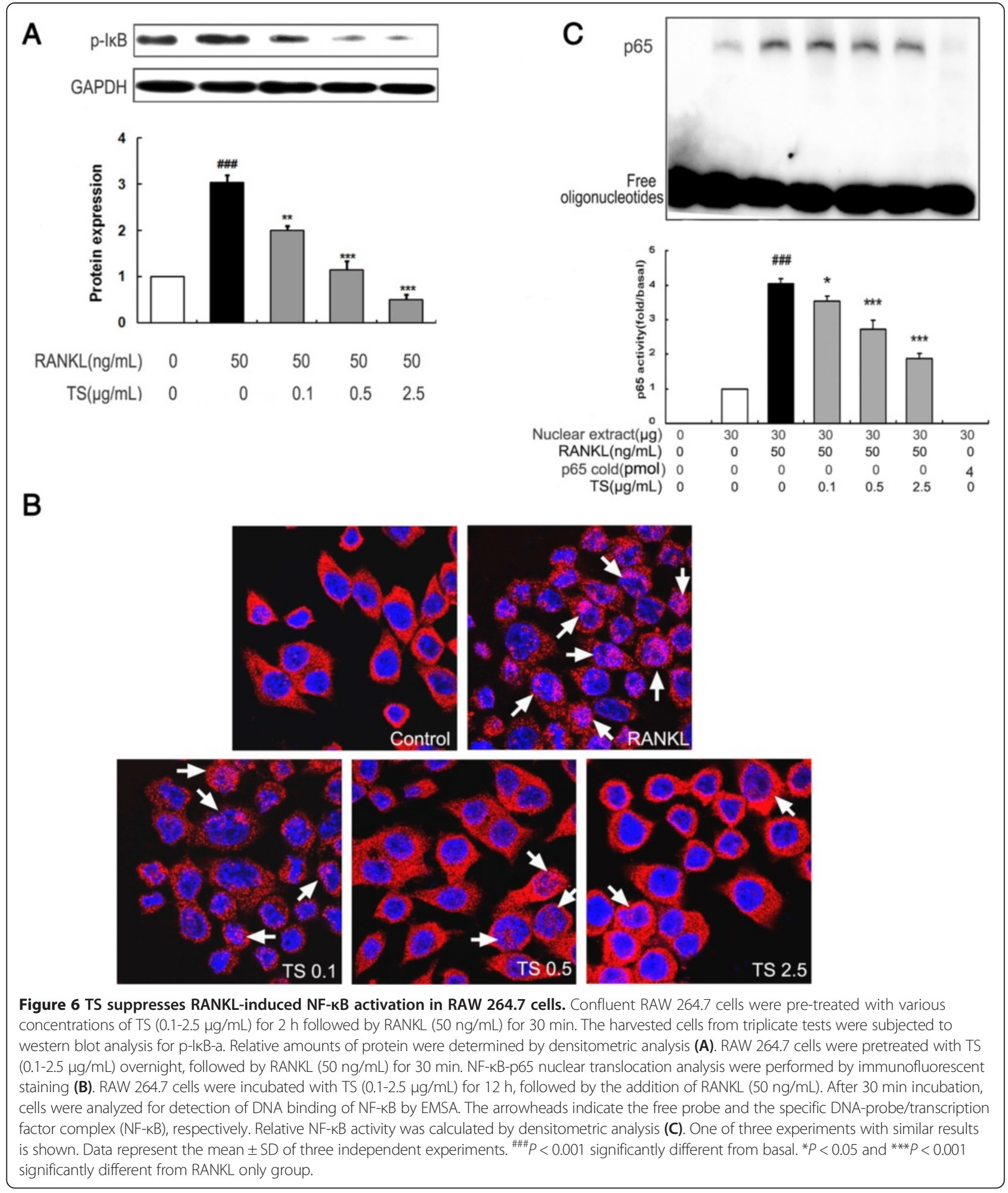

provide the mechanistic explanation, at least in part for the protective effect of TS against lytic bone diseases, such as in RA.

Recent studies have identified osteoclasts as the principal cell type responsible for bone destruction [24].
Osteoclasts are unique multinuclear giant cells, which are derived from monocyte-macrophage lineage cells. Their differentiation from precursors can be induced by RANKL, a key factor that also controls the function and survival of mature osteoclasts [25]. As a ligand, RANKL 


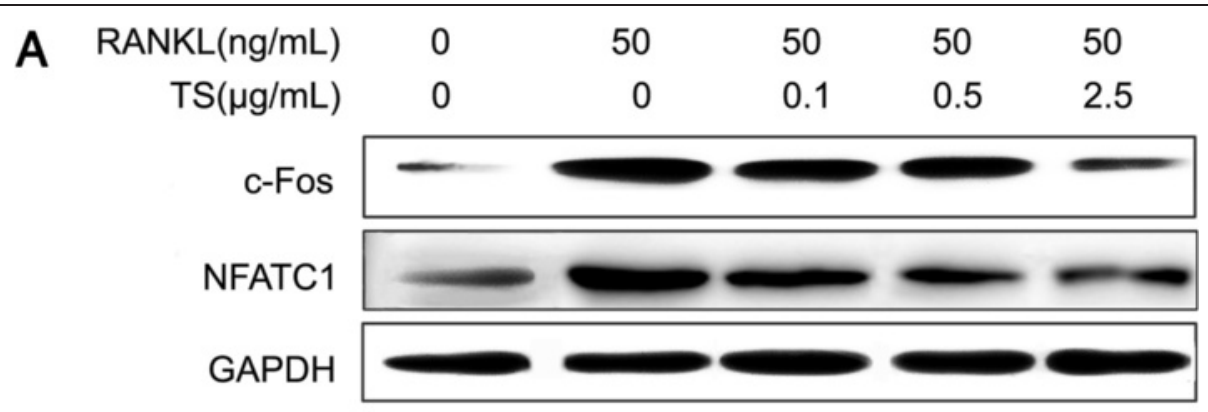

B

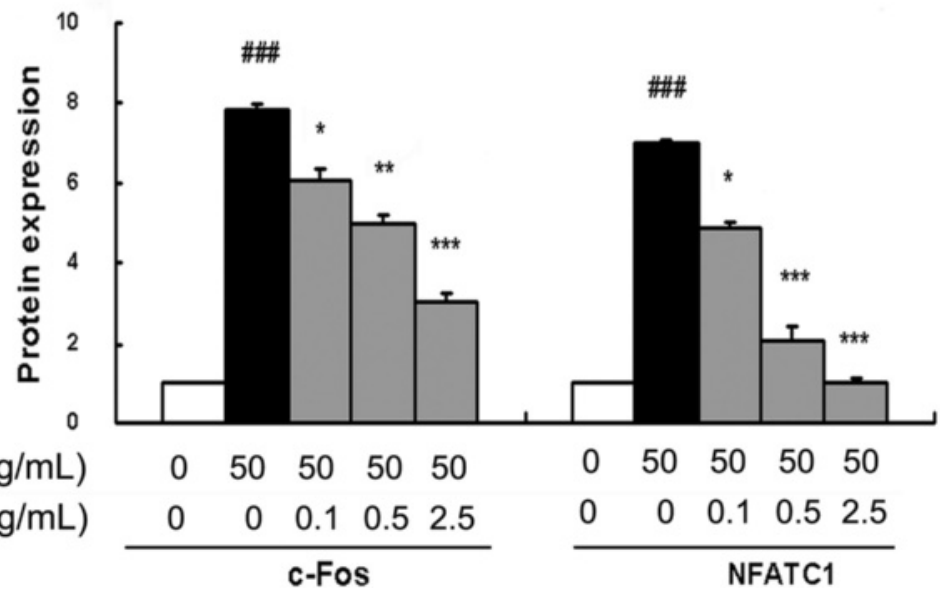

Figure 7 TS down-regulates RANKL-induced expression of NFTAc1 and c-Fos in RAW 264.7 cells. RAW 264.7 cells were preincubated with indicated concentrations of TS for $2 \mathrm{~h}$, and then treated with $50 \mathrm{ng} / \mathrm{mL}$ RANKL for $24 \mathrm{~h}$. Total protein was extracted and subjected to Western blot analysis using antibodies against NFATC1 and c-Fos (A). Relative amounts of each protein were determined by densitometric analysis (B). One of three experiments with similar results is shown. Data represent the mean \pm SD of three independent experiments. \#\#\# $P<0.001$ significantly different from basal. ${ }^{*} P<0.05,{ }^{*} P<0.01$ and ${ }^{* * *} P<0.001$ significantly different from RANKL only group.

interacts to its receptor RANK, and induces them to differentiate into osteoclasts. Notably, until now, no other endogenous factors that can lead to osteoclast formation without RANKL participation have been found. Therefore, RANKL is used as a convincing inducer in studies on differentiation and function of osteoclasts. In the present study, we adopted the RANKL-induced osteoclast differentiation platform from RAW 264.7 cells, and our results revealed that TS significantly reduced the osteoclast differentiation. It is generally accepted that the formation of bone resorption pits occurs in conjunction with the process of osteoclast differentiation. In addition, the results in our study showed that TS dramatically attenuates osteoclastic bone resorption as detected by bone resorption pit assay. These data confirmed that TS inhibits both the formation and the resorptive function of osteoclasts.

Inflammatory cytokines, such as TNF-a, induce preosteoclast fusion, support the survival of mature osteoclasts, and stimulate osteoclastic bone resorption [21]. It was recently reported that the RANK-RANKL pathway is involved in TNF-a-induced osteoclast dif- ferentiation [26-29]. Triterpenoid saponins from $A$ flaccida have previously been reported to inhibit LPSinduced cyclooxygenase-2, and PGE2 expression in BEL-7402 and HepG2 hepatoma cell [15]. In the present study, TS strongly suppressed RANKL-induced TNF-a secretion by RAW 264.7 cells, which partly explains the application of TS in diseases with inflammatory bone destruction.

RANKL stimulates osteoclast precursors to commit to the osteoclastic phenotype by binding to its receptor RANK on the surface of osteoclast precursors [7]. The binding of RANKL with its receptor RANK interacts and induces the trimerization and activation of signaling adaptor molecule TRAF6, which is an essential initiating step during osteoclast differentiation [9]. Our data showed that TS significantly inhibited TRAF6 expression. As downstream of RANK signaling, three major subfamilies of MAPKs (ERK 1/2, JNK and p38 MAPK) have been implicated as key regulators of various cellular responses, including cell proliferation, differentiation and apoptosis [30,31]. These MAPKs also play pivotal roles in the development of osteoclasts [32-34], and are thus key 
molecular targets for therapeutic application in inflammatory bone diseases [35]. Indeed, specific inhibitors for ERK1/2, JNK and p38 MAPK could disturb RANKLstimulated osteoclast differentiation [22,36-38]. In the present study, we demonstrated that TS could notably inhibit the RANKL-induced phosphorylation of JNK and p38 MAPK, suggesting that TS targets JNK and p38 MAPK cascades.

Genetic studies have demonstrated that NF-kB signaling pathway also plays a crucial role in osteoclast differentiation [11]. NF- $\mathrm{kB}$ knockout mice showed the defects of osteoclast differentiation and severe osteopetrosis, indicating that NF- $\mathrm{kB}$ is a crucial factor in osteoclast differentiation [39]. The classical NF- $k B$ signaling pathway involves the activation of the IKK complex, which phosphorylates $\mathrm{I} K \mathrm{~B} \alpha$ and targets them for ubiquitin-dependent degradation [9]. In the alternative IкB-independent pathway, direct phosphorylation of NF-kB subunit p65 by IKK also modulates NF- $\mathrm{KB}$ transcription activity [40]. In this study, TS inhibited RANKL-induced NF- $\mathrm{BB}$ activation by inhibiting $\mathrm{p}-\mathrm{I} \kappa \mathrm{B} \alpha$ and $\mathrm{p} 65$ nuclei translocation and DNA binding activity, which indicates that inhibiting the activation of NF- $\mathrm{kB}$ pathway contributed to the suppression of TS against osteoclast differentiation.

Following the activation of the MAPK and NF- $\mathrm{kB}$ signaling pathways caused by RANKL, multiple osteoclastogenic transcription factors, such as c-Fos, NFATc1 and PU.1, should be induced to mediate the formation of mature and functionally active osteoclasts [41]. When BMMs are stimulated by RANKL, the expression of NFATc1 is induced through c-Fos and auto amplification by NFATc1 [41,42]. NFATc1-deficient embryonic stem cells do not form mature osteoclasts by RANKL treatment and overexpression of ectopic ca-NFATc1 in BMMs appropriately induces osteoclast differentiation from BMMs even in the absence of RANKL [41,43]. c-Fos, a major component of the transcription factor AP-1, can induce the expression of NFATc1 to regulate osteoclast differentiation after RANKL stimulation. Mice lacking c-Fos develop osteopetrosis as a result of a complete ablation of osteoclast formation. Our results revealed that RANKL stimulation markedly up-regulated the expression of both c-Fos and NFATc1, which is dramatically suppressed by TS treatment.

\section{Conclusions}

In conclusion, our results revealed for the first time that TS could suppress RANKL-induced osteoclast differentiation in vitro. We also clarified that the inhibitory effects of TS occur via the down-regulation of TRAF6 level, suppression of JNK, p38 MAPKs and NF- $\mathrm{kB}$ activation, subsequent decreased expression of c-Fos and NFATc1 (summarized in Figure 8). These results highlight the antiosteoclast differentiation potential of TS, and provide

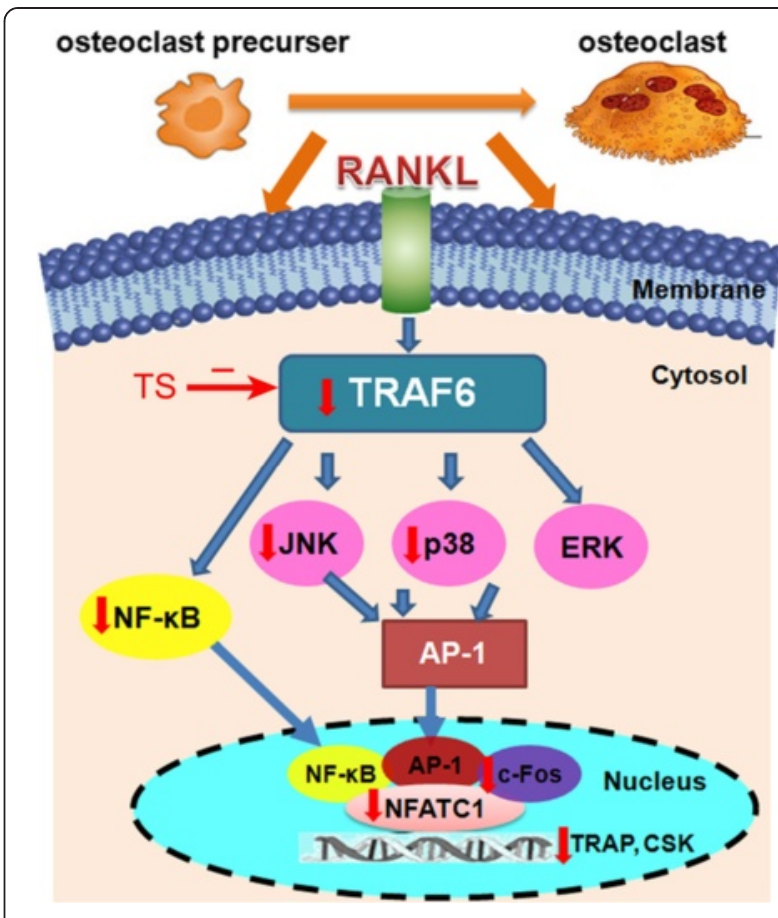

Figure 8 Schematic model for TS regulation on the osteoclast differentiation and activation. The figure summarizes the results presented with this study. Vertical arrows indicate that an inhibition or a downregulation in osteoclast was observed.

further mechanism of TS in the application of RA. Furthermore, TS may be a potential agent and needs to be more evaluated in vivo or in clinical trials to become a therapeutic for the lytic bone diseases, especially inflammatory bone destruction.

\section{Additional files}

Additional file 1: Figure S1. TS has no effect on BMMS viability. BMMs were treated with TS for $48 \mathrm{~h}$, and cell viability was measured by a MTS viability assay. Data represent the mean \pm SD of three independent experiments.

Additional file 2: Figure S2. TS shows no effect on cell viability. RAW 264.7 cells were incubated with indicated concentrations of TS for $24 \mathrm{~h}$, and then tested by trypan blue dye exclusion. Data represent the mean \pm SD of three independent experiments.

Additional file 3: Figure S3. TS treatment has no effect on cell apoptosis. RAW 264.7 cells were cultured in the presence of TS for $48 \mathrm{~h}$ and stained with Hoescht 33258, and analyzed with fluorescence microscope. Cells with nuclei containing condensed chromatin or cells with fragmented nuclei were defined as apoptotic cells. Magnification $600 x$.

\section{Competing interests}

The authors declare that they have no competing interests.

\section{Authors' contributions}

XYK, WBW, YY, WHY, XML, MCZ and XHS carried out experimental analysis and acquisition of data, analysis and interpretation of the data. SWJ, HYZ and DHJ contributed reagents/osteoclast staining technique; XYK and NL conceived the study, participated in its design and coordination, obtained funding, and drafted the manuscript. All authors read and approved the final manuscript. 


\section{Acknowledgements}

This study was supported by grants from the major projects of national science and technology (2011ZX09101-006-03,2013ZX09301307), natural science foundation of Beijing (No. 7112096), and fundamental research funds for the central public welfare research institutes (ZZ070836).

\section{Author details}

'Institute of Chinese Materia Medica, China Academy of Chinese Medical Sciences, No. 16, Nanxiaojie, Dongzhimennei, Beijing 100700, China. ${ }^{2}$ Institute of Basic Theory, China Academy of Chinese Medical Sciences, Beijing 100700, China. ${ }^{3}$ Guangzhou Kanghe Pharmaceutical Limited Company, Guangzhou 511440, China

\section{Received: 25 September 2014 Accepted: 17 February 2015}

Published online: 15 March 2015

\section{References}

1. Matsuo K. Cross-talk among bone cells. Curr Opin Nephrol Hypertens. 2009;18:292-7.

2. Boyle WJ, Simonet WS, Lacey DL. Osteoclast differentiation and activation. Nature. 2003;423:337-42.

3. Cully M. Rheumatoid arthritis: Uncoupling bone and cartilage destruction. Nat Rev Rheumatol. 2013;9:446.

4. Ochi S, Shinohara M, Sato K, Gober HJ, Koga T, Kodama T, et al. Pathological role of osteoclast costimulation in arthritis-induced bone loss. Proc Natl Acad Sci U S A. 2007;104:11394-9.

5. Broadhead ML, Clark JC, Dass CR, Choong PF, Myers DE. Therapeutic targeting of osteoclast function and pathways. Expert Opin Ther Targets. 2011;15:169-81.

6. Kukita A, Kukita T, Nagata K, Teramachi J, Li YJ, Yoshida H, et al. The transcription factor FBI-1/OCZF/LRF is expressed in osteoclasts and regulates $R A N K L$-induced osteoclast formation in vitro and in vivo. Arthritis Rheum. 2011:63:2744-54

7. Lacey DL, Timms E, Tan HL, Kelley MJ, Dunstan CR, Burgess T, et al. Osteoprotegerin ligand is a cytokine that regulates osteoclast differentiation and activation. Cell. 1998:93:165-76.

8. Gohda J, Akiyama T, Koga T, Takayanagi H, Tanaka S, Inoue J. RANK-mediated amplification of TRAF6 signaling leads to NFATC1 induction during osteoclastogenesis. EMBO J. 2005;24:790-9.

9. Asagiri $\mathrm{M}$, Takayanagi $\mathrm{H}$. The molecular understanding of osteoclast differentiation. Bone. 2007:40:251-64

10. Bai S, Zha J, Zhao H, Ross FP, Teitelbaum SL. Tumor necrosis factor receptor-associated factor 6 is an intranuclear transcriptional coactivator in osteoclasts. J Biol Chem. 2008:283:30861-7.

11. Takayanagi H. Osteoimmunology: shared mechanisms and crosstalk between the immune and bone systems. Nat Rev Immunol. 2007;7:292-304.

12. Banu J, Varela E, Fernandes G. Alternative therapies for the prevention and treatment of osteoporosis. Nutr Rev. 2012:70:22-40.

13. Han LT, Li J, Huang F, Yu SG, Fang NB. Triterpenoid saponins from Anemone flaccida induce apoptosis activity in HeLa cells. J Asian Nat Prod Res. 2009;11:122-7.

14. Bing $F$, Zhang $G$. The national five kind of new medicine-rhizome of Anemone Flaccid Fengshi'an capsule. Hubei J Tradit Chin Med. 2005:27:48-9.

15. Han LT, Fang Y, Li MM, Yang HB, Huang F. The Antitumor Effects of Triterpenoid Saponins from the Anemone flaccida and the Underlying Mechanism. Evid Based Complement Alternat Med. 2013;2013:517931.

16. Nakashima T, Takayanagi H. Osteoimmunology: crosstalk between the immune and bone systems. J Clin Immunol. 2009:29:555-67.

17. Jung SM, Kim KW, Yang CW, Park SH, Ju JH. Cytokine-mediated bone destruction in rheumatoid arthritis. J Immunol Res. 2014:2014:263-5.

18. Kong X, Liu C, Zhang C, Zhao J, Wang J, Wan H, et al. The suppressive effects of Saposhnikovia divaricata (Fangfeng) chromone extract on rheumatoid arthritis via inhibition of nuclear factor-kappaB and mitogen activated proteinkinases activation on collagen-induced arthritis model. J Ethnopharmacol. 2013;148:842-50.

19. Hsu H, Lacey DL, Dunstan CR, Solovyev I, Colombero A, Timms E, et al. Tumor necrosis factor receptor family member RANK mediates osteoclast differentiation and activation induced by osteoprotegerin ligand. Proc Nat Acad Sci U S A. 1999:96:3540-5.
20. Griese JJRPJ, Schwarzinger S, Dobbek H. Xenobiotic reductase A in the degradation of quinoline by Pseudomonas putida 86: physiological function, structure and mechanism of 8-hydroxycoumarin reduction. J Mol Biol. 2006;361:140-52.

21. Huang QQ, Pope RM. The role of toll-like receptors in rheumatoid arthritis. Curr Rheumatol Rep. 2009;11:357-64.

22. Cheng B, Li J, Du J, Lv X, Weng L, Ling C. Ginsenoside Rb1 inhibits osteoclastogenesis by modulating NF-kappaB and MAPKs pathways. Food Chem Toxicol. 2012;50:1610-5.

23. Bing F, Zhang G, Deng C, Gong Y. Immune mechanisms of Wugong Sanqi extract against CIA in mouse. Chin J Immunol. 2008;24:716-20.

24. Braun T, Zwerina J. Positive regulators of osteoclastogenesis and bone resorption in rheumatoid arthritis. Arthritis Res Ther. 2011;13:235.

25. Takayanagi H, Kim S, Matsuo K, Suzuki H, Suzuki T, Sato K, et al. RANKL maintains bone homeostasis through c-Fos-dependent induction of interferon-beta. Nature. 2002;416:744-9.

26. Zou W, Bar-Shavit Z. Dual modulation of osteoclast differentiation by lipopolysaccharide. J Bone Miner Res. 2002;17:1211-8.

27. Siggelkow H, Eidner T, Lehmann G, Viereck V, Raddatz D, Munzel U, et al Cytokines, osteoprotegerin, and RANKL in vitro and histomorphometric indices of bone turnover in patients with different bone diseases. J Bone Miner Res. 2003;18:529-38.

28. Lubberts E, Koenders MI, Oppers-Walgreen B, van den Bersselaar L, Coenen-de Roo CJ, Joosten LA, et al. Treatment with a neutralizing anti-murine interleukin-17 antibody after the onset of collagen-induced arthritis reduces joint inflammation, cartilage destruction, and bone erosion. Arthritis Rheum. 2004;50:650-9.

29. Yago T, Nanke Y, Ichikawa N, Kobashigawa T, Mogi M, Kamatani N, et al. IL-17 induces osteoclastogenesis from human monocytes alone in the absence of osteoblasts, which is potently inhibited by anti-TNF-alpha antibody: a novel mechanism of osteoclastogenesis by IL-17. J Cell Biochem. 2009;108:947-55.

30. Kim HK, Kim MG, Leem KH. Osteogenic Activity of Collagen Peptide via ERK/MAPK Pathway Mediated Boosting of Collagen Synthesis and Its Therapeutic Efficacy in Osteoporotic Bone by Back-Scattered Electron Imaging and Microarchitecture Analysis. Molecules. 2013;18:15474-89.

31. Sui X, Kong N, Ye L, Han W, Zhou J, Zhang Q, et al. p38 and JNK MAPK pathways control the balance of apoptosis and autophagy in response to chemotherapeutic agents. Cancer Lett. 2014:344:174-9.

32. Li C, Yang Z, Li Z, Ma Y, Zhang L, Zheng C, et al. Maslinic acid suppresses osteoclastogenesis and prevents ovariectomy-induced bone loss by regulating RANKL-mediated NF-kappaB and MAPK signaling pathways. J Bone Miner Res. 2011:26:644-56.

33. Huh JE, Jung IT, Choi J, Baek YH, Lee JD, Park DS, et al. The natural flavonoid galangin inhibits osteoclastic bone destruction and osteoclastogenesis by suppressing NF-kappaB in collagen-induced arthritis and bone marrowderived macrophages. Eur J Pharmacol. 2013;698:57-66.

34. Zhai ZJ, Li HW, Liu GW, Qu XH, Tian B, Yan W, et al. Andrographolide suppresses RANKL-induced osteoclastogenesis in vitro and prevents inflammatory bone loss in vivo. Br J Pharmacol. 2014;171:663-75.

35. Stevenson DA, Schwarz EL, Carey JC, Viskochil DH, Hanson H, Bauer S, et al. Bone resorption in syndromes of the Ras/MAPK pathway. Clin Genet. 2011;80:566-73.

36. Ikeda F, Matsubara T, Tsurukai T, Hata K, Nishimura R, Yoneda T. JNK/c-Jun signaling mediates an anti-apoptotic effect of RANKL in osteoclasts. J Bone Miner Res. 2008;23:907-14.

37. Tao H, Okamoto M, Nishikawa M, Yoshikawa H, Myoui A. P38 mitogenactivated protein kinase inhibitor, FR167653, inhibits parathyroid hormone related protein-induced osteoclastogenesis and bone resorption. PLoS One. 2011;6:e23199

38. Yamanaka Y, Clohisy JC, Ito H, Matsuno T, Abu-Amer Y. Blockade of JNK and NFAT pathways attenuates orthopedic particle-stimulated osteoclastogenesis of human osteoclast precursors and murine calvarial osteolysis. J Orthop Res. 2013;31:67-72.

39. Leibbrandt A, Penninger JM. RANKL/RANK as key factors for osteoclast development and bone loss in arthropathies. Adv Exp Med Biol. 2009;649:100-13.

40. Sakurai $H$, Chiba H, Miyoshi H, Sugita T, Toriumi W. IkappaB kinases phosphorylate NF-kappaB p65 subunit on serine 536 in the transactivation domain. J Biol Chem. 1999:274:30353-6.

41. Takayanagi H, Kim S, Koga T, Nishina H, Isshiki M, Yoshida H, et al. Induction and activation of the transcription factor NFATC1 (NFAT2) integrate RANKL signaling in terminal differentiation of osteoclasts. Dev Cell. 2002;3:889-901. 
42. Asagiri M, Sato K, Usami T, Ochi S, Nishina H, Yoshida H, et al.

Autoamplification of NFATC1 expression determines its essential role in bone homeostasis. J Exp Med. 2005;202:1261-9.

43. Lee SH, Kim T, Jeong D, Kim N, Choi Y. The tec family tyrosine kinase Btk Regulates RANKL-induced osteoclast maturation. J Biol Chem. 2008:283:11526-34.

Submit your next manuscript to BioMed Central and take full advantage of:

- Convenient online submission

- Thorough peer review

- No space constraints or color figure charges

- Immediate publication on acceptance

- Inclusion in PubMed, CAS, Scopus and Google Scholar

- Research which is freely available for redistribution 